DOI: 10.1590/permusi2015a3103

\title{
Gesto na performance da percussão, Parte 2: análise fatorial de correspondências de dados experimentais ${ }^{1}$
}

\author{
Fernando Chaib (N三P3/IFG² - Inst. Fed. Ed., Ciência e Tecnologia, Goiânia, GO) \\ fernandochaib@gmail.com
}

Homero Chaib Filho (N三P/IFG³ - Inst. Fed. Ed., Ciência e Tecnologia, Goiânia, GO) homerochaibfilho@gmail.com

\author{
João Catalão (Slxtrum/Université de Montreal, Montreal, Canadá) \\ catalao@ktzperc.com
}

\begin{abstract}
Resumo - Este artigo, Parte 2 do mesmo estudo, apresenta os resultados do experimento "Sensação de continuidade de um trecho musical" (CHAIB et. al. 2013) analisados através da técnica estatística Análise Fatorial de Correspondências (AFC). O objetivo principal dessa análise é o de observar, através de índices, a influência do gesto na transmissão de sensações de continuidade, suspensão e conclusão sobre a performance em percussão. Uma vez alcançado o objetivo será possível generalizar os resultados em performances realizadas sobre os mesmos moldes das apresentadas no experimento.
\end{abstract}

Palavras-chave: influência do gesto na Performance; percussão; AFC; sensações na performance.

\section{Gesture on percussion performance, Part 2: correspondence factorial analysis of experimental data}

\begin{abstract}
This paper, Part 2 of the same study, presents the results of the experiment "Sensation of continuity of a musical excerpt" (CHAIB et. al. 2013) analyzed using the statistical technique Factor Analysis of Correspondences (FAC). The main objective of this analysis is to look through indexes, the influence of gesture in conveying sensations of continuity, suspension and conclusion on the performance on percussion. Once achieved the goal will be possible to generalize the results in performances conducted on the same lines presented in the experiment.
\end{abstract}

Keywords: gesture influence in performance; percussion; FAC; sensations in performing.

1. Introdução - Depois de constatada por nós a influência dos gestos corporais sobre as sensações de continuidade, suspensão e conclusão de um individuo, em uma performance percussiva em específico (CHAIB et. al. 2013), procuramos evidenciar a influência dos gestos sobre essas mesmas sensações correlacionando os estímulos ${ }^{4}$ com os gestos ${ }^{5}$ e Fatores Contextuais ${ }^{6}$ desenvolvidos para o experimento "Sensação de continuidade de um trecho musical" (CHAIB et. al. 2013). Para isso, utilizamos os dados colhidos nesse experimento para a realização da Análise Fatorial de Correspondências (AFC).

Neste artigo será possível perceber que ao realizar-se uma performance em percussão nos moldes apresentados no experimento em questão, os níveis de influência dos gestos sobre essas performances em percussão poderão manter-se relacionados. Isso poderá trazer, ao intérprete percussionista, uma série de reflexões capazes de revolucionar a sua relação com o corpo e performance, sempre obviamente em função da música executada. 
2. Sobre o Experimento "Sensação de continuidade de um trecho musical"7 - $O$ experimento significou a apresentação de três trechos subsequentes da obra Exil: Shangai 45, de Michel Longtin, gravados sobre os estímulos áudio (A), visual (V) e audiovisual (AV) para três grupos de dois indivíduos cada. Dois tipos de gestos foram utilizados para a gravação dos estímulos: Gesto Técnico (GT) e Gesto Expressivo (GE). Através da geração de um PATCH desenvolvido com o software MAX MSP 6.0, os participantes exprimiram suas sensações de continuidade, suspensão e conclusão, utilizando teclas coloridas no computador. Cada grupo de indivíduos apreciou um estímulo combinado com um tipo de gesto e trecho diferentes (Ex.1) ${ }^{8}$.

\begin{tabular}{|l|l|l|}
\hline $\begin{array}{l}\text { In.1 e In.2 } \\
\text { Gr. I }\end{array}$ & $\begin{array}{l}\text { In.1 e In.2 } \\
\text { Gr. II }\end{array}$ & $\begin{array}{l}\text { In.1 e In.2 } \\
\text { Gr. III }\end{array}$ \\
\hline A1t & A2t & A3t \\
\hline A3e & A1e & A2e \\
\hline V2t & V3t & V1t \\
\hline V1e & V2e & V3e \\
\hline AV3t & AV1t & AV2t \\
\hline AV2e & AV3e & AV1e \\
\hline
\end{tabular}

Ex.1 - Divisão dos Estímulos por grupos, onde: In. (Indivíduo), Gr. (grupo).

Para o reconhecimento das respostas no programa e formulação das tabelas a serem utilizadas na AFC, determinamos a letra $\boldsymbol{a}$ como sensação de continuidade; a letra $\boldsymbol{b}$ sensação de suspensão e $\boldsymbol{d}$, consequentemente, conclusão. Combinados estímulos, gestos e trechos, obtivemos 18 tipos de apresentações distintas gerando, ao todo, 36 respostas. Dessas 36 respostas gerou-se uma tabela de contingência (Ex.2) utilizada para a AFC.

3. Sobre a AFC - Embora estejamos lidando com questões subjetivas latentes que vem de encontro com estados emocionais e comportamentais do espectador - uma vez que, segundo SANTIAGO e MEYEREWICZ (2009, p.85), o gesto "também é portador da identidade cultural do instrumentista, envolvendo seus valores, costumes e comportamentos vivenciados socialmente" - o tipo de resposta apresentada pelo experimento realizado conduz a uma tabela de dados que nos levou a optar por trabalhar com uma técnica de análise fatorial:

\begin{abstract}
Dado que el problema de la medida en las ciencias sociales y del comportamiento adquiere especial dificultad, estos métodos, que relajan los requisitos exigibles a los datos, abren un amplio espectro de posibilidades para el estudio de estos fenómenos. La simplicidad de la interpretación de los resultados, junto con la disponibilidad de paquetes estadísticos de fácil manejo, hacen de esta técnica un instrumento imprescindible para cualquier investigador (BATISTA e SUREDA, 1987, p.183).
\end{abstract}

Quando empregamos este tipo de análise estamos interessados no comportamento de uma variável ou grupos de variáveis em covariação com outras. As técnicas de análise multivariada são úteis para descobrimos regularidades no comportamento de duas ou mais variáveis e para testar modelos alternativos de associação entre tais variáveis, incluindo a determinação de quando e como dois ou mais grupos diferem em seu perfil multivariado. Isso nos permitirá admitir que a relação estatística obtida no experimento 
poderá refletir-se sobre outros eventos envolvendo a performance para percussão, que não apenas os aqui reproduzidos.

A Análise Fatorial é uma das técnicas mais usuais do que se convencionou chamar Análise Multivariada. Tais técnicas visam ordenar e reduzir o número de variáveis correlacionadas entre si, através da geração de fatores ${ }^{9}$, que associados aos autovalores denominam-se componentes principais, permitindo a explicação da variabilidade do conjunto de dados iniciais (BARROSO, 2003). A análise fatorial é essencialmente um método para determinar os fatores principais existentes em um conjunto de dados, definindo quais indivíduos ou variáveis pertencem as quais fatores, e com qual intensidade de pertinência tais indivíduos ou variáveis ocorrem em um fator. Existem duas vertentes essenciais para essas técnicas: uma que se baseia na estatística inferencial (abordagem anglo-saxônica); outra que se baseia na estatística descritiva (abordagem francesa). Para diferenciar, os que se utilizam da abordagem francesa costumam chamar essas técnicas de multidimensionais.

A AFC, uma das técnicas de análise multidimensional ${ }^{10}$, é, por assim dizer, uma evolução da Análise de Componentes Principais (ACP) dirigida para a análise tabelas de dados que representam contagem (nessa categoria não se enquadram dados contínuos como valores correspondentes a medidas de peso, altura, tempo, etc.) e que são dados discretos (número de vezes que se aperta uma determinada tecla, por exemplo). Na AFC, costuma-se representar a tabela de dados como uma matriz $n x m$ onde $\mathrm{n}$ é o número de variáveis e $\mathrm{m}$ o número de indivíduos. O conteúdo da matriz (ou tabela) é o número de ocorrências de um indivíduo em uma variável e a ela dá-se o nome de tabela de contingência.

Desta forma a AFC gera fatores que agregam as variáveis ou indivíduos com seus respectivos graus de pertinência. Tais fatores têm uma hierarquia: o, assim designado, primeiro fator é o que retém a maior quantidade de informações (ou o de maior peso das informações) contida na tabela de contingência; o segundo fator retém a segunda maior quantidade de informação; o terceiro, a terceira maior quantidade, e assim por diante. Esses fatores são, na verdade, eixos que definem espaços bidimensionais, tridimensionais, quadridimensionais e n-dimensionais. $O$ plano é um espaço bidimensional e cada par de fatores gerados forma um plano. Aquele composto pelos dois primeiros fatores é o que agrega a maior parte das informações contidas na tabela de dados e chama-se primeiro plano fatorial e é sobre o qual, em geral, que se atém a atenção de análise. Enquanto resultado, poderemos, através dos fatores, observar como as variáveis ou os indivíduos se relacionam; e no plano como variáveis e indivíduos se relacionam em relação aos próprios fatores.

Os dados obtidos do experimento receberam devido tratamento para permitir a montagem de uma tabela de contingência (Ex.2) com vistas à realização da Análise Fatorial de Correspondências (AFC), onde:

- as linhas correspondem, através das execuções técnica $(\mathrm{t})$ ou expressiva (e), aos estímulos fornecidos no experimento realizado: auditivo ( $\mathbf{A t}$ e $\mathbf{A e}$ ), visual (Vt e Ve) ou audiovisual (AVt e AVe).

- as colunas correspondem às sensações de continuidade (a), suspensão (b) e conclusão (d) em cada Fator Contextual (F). 
Desta forma, cada célula da tabela corresponde ao número de respostas dadas à uma sensação dentro de um fator contextual devido a um estímulo. A execução da AFC foi feita sobre os dados dessa tabela utilizando-se o software Statistical Analysis System (SAS) da Empresa Brasileira de Pesquisa Agropecuária (EMBRAPA), e os resultados estão discutidos a seguir. Para facilitar a execução do SAS ao invés de cores, definiram-se as seguintes letras para os tipos de sensação dentro de cada fator contextual: $a=$ continuidade, $b=$ suspensão e $d=$ conclusão. Sendo assim, as colunas da tabela, para execução do software, serão nominadas como: F1a, F1b, F1d, F2a, F2b, e assim por diante até F8d. Desta forma, como exemplo, F1a representa a sensação de continuidade para o Fator Contextual F1 devido a algum estímulo. Semelhantemente, F4d representará a sensação de conclusão no Fator Contextual F4.

A partir dos pontos definidos pela tabela de contingência (Ex.2) e através de combinações lineares dessa tabela, a AFC concebe fatores que geram um espaço vetorial, no qual os pontos da tabela são projetados ${ }^{11}$. Cada fator gerado retém uma quantidade de informação (associada ao peso relativo que os pontos possuem uns em relação aos outros na tabela de contingência) de maneira a que a soma da quantidade de informação de todos os fatores é igual a quantidade de informação total contida na tabela inicial.

Os fatores (também chamados eixos fatoriais) com maior quantidade de informação, são chamados "fatores principais", e são ordenados, decrescentemente, pela ordem de importância (ou peso, igual a quantidade de informação retida pelo eixo fatorial). Os dois primeiros eixos fatoriais (os de maior peso), geram o primeiro plano fatorial que é o que retém maior quantidade de informação com respeito aos dados iniciais. Busca-se, então, um plano fatorial que retenha uma quantidade expressiva da informação total e considera-se que a partir de $70 \%$ da informação total, existe uma quantidade boa para a realização da análise e estabelecimento das relações que extraem as informações dos dados da tabela de contingência.

Ao executarmos a AFC sobre a tabela equivalente ao Ex.2, observamos que o primeiro plano fatorial (como dito acima, definido pelos dois primeiros eixos fatoriais) reteve aproximadamente $74 \%$ da informação total, sendo que o primeiro eixo reteve $42,73 \%$ e o segundo $30,74 \%$ da informação total (Ex.3).

Consideramos, assim, que o primeiro plano fatorial reteve informações suficientes para a caracterização dos eixos fatoriais e o estabelecimento de relações inter e entre as linhas (os estímulos) e as colunas (as sensações para cada fator contextual). Para criarmos condições de estabelecer tais relações faremos uma tipificação dos dois primeiros eixos em relação aos estímulos (linhas), podendo também ser tipificado em relação às sensações (colunas). Ressaltamos que o uso do termo "importância para a formação de um eixo" se refere à caracterização com fins para a tipificação. Essa tipificação é que permitirá a generalização dos resultados do experimento realizado. 


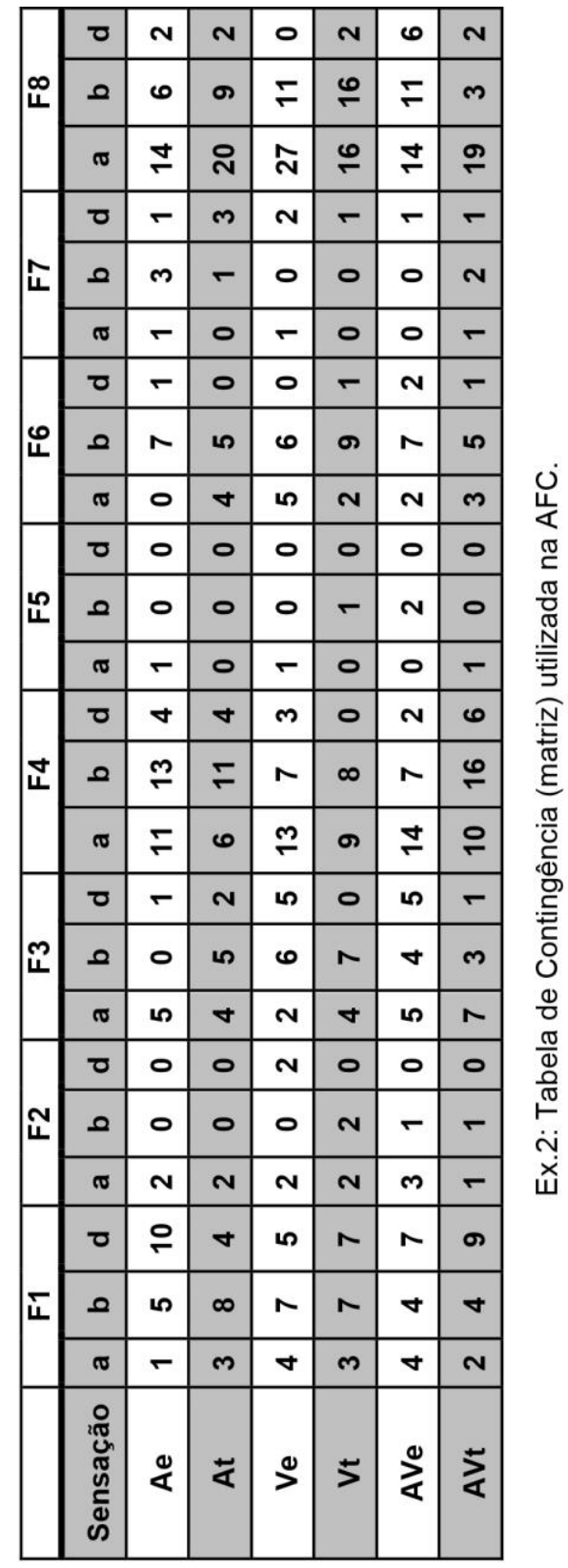

Inertia and Chi-Square Decomposition

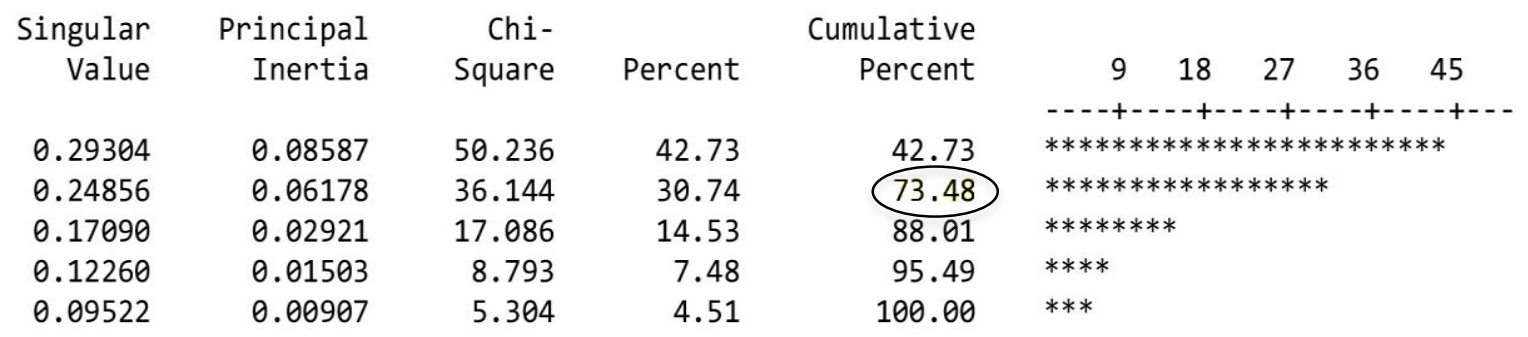

Ex.3 - Primeiro plano fatorial. 


\section{4 - Tipificação dos eixos fatoriais pelas linhas ${ }^{12}$ (estímulos)}

Estudaremos a contribuição relativa (peso associado à quantidade de informação para a geração de um fator) de cada estímulo para a formação dos dois primeiros eixos fatoriais. Será importante conhecer, também, a maneira como esses estímulos se correlacionam. Para isso devemos observar o sinal (+ ou -) de suas coordenadas no plano (Ex.4):

\begin{tabular}{|l|l|}
\hline \multicolumn{2}{|l|}{ Contribuição Parcial } \\
\hline Estímulo & Eixo 1 \\
\hline Ae & $\mathbf{3 3 , 5 9}$ \\
\hline AVt & $\mathbf{3 1 , 1 8}$ \\
\hline Vt & $\mathbf{1 6 , 2 8}$ \\
\hline $\mathrm{Ve}$ & 9,52 \\
\hline $\mathrm{AVe}$ & 8,81 \\
\hline $\mathrm{At}$ & 0,62 \\
\hline
\end{tabular}

\begin{tabular}{|l|l|}
\hline \multicolumn{2}{|l|}{ Contribuição Parcial } \\
\hline Estímulo & Eixo 2 \\
\hline Ve & $\mathbf{3 7 , 1 2}$ \\
\hline $\mathbf{A V e}$ & $\mathbf{3 2 , 8 1}$ \\
\hline $\mathrm{At}$ & $\mathbf{1 6 , 3 0}$ \\
\hline $\mathrm{Vt}$ & 10,59 \\
\hline $\mathrm{Ae}$ & 3,05 \\
\hline $\mathrm{AVt}$ & 0,12 \\
\hline
\end{tabular}

\begin{tabular}{|l|l|l|}
\hline \multicolumn{3}{|c|}{ Sinal das Coordenadas } \\
\hline Estímulo & Eixo 1 & Eixo 2 \\
\hline At & - & - \\
\hline Vt & - & + \\
\hline AVt & + & - \\
\hline Ae & + & + \\
\hline Ve & - & - \\
\hline AVe & - & + \\
\hline
\end{tabular}

Ex..4 - Contribuição parcial das linhas (estímulos) para a formação do $1^{\circ}$ e $2^{\circ}$ fatores (eixos); sinais das coordenadas nos eixos fatoriais.

Vê-se que os estímulos que mais contribuíram para a formação do primeiro eixo (denominado Eixo 1 no Ex.4) foram Ae, AVt e Vt, nesta ordem de importância. Suas coordenadas possuem nesse eixo sinal positivo para os dois primeiros estímulos e negativo para o terceiro. Assim, constatamos que, no que diz respeito ao primeiro fator, existe correlação positiva entre os estímulos Ae e AVt e esses dois estímulos têm correlação negativa com o estímulo Vt.

Lembramos que uma correlação positiva entre duas variáveis implica na mesma forma de variação entre elas: se uma aumenta a outra aumenta também; se uma diminui a outra também diminuirá. Já a correlação negativa implica em variações contrárias: se uma aumenta a outra diminuirá e vice-versa.

No nosso caso, diremos que o estímulo Ae provoca reação semelhante ao estímulo AVt e que o estímulo Vt provoca reação inversa aos dois anteriores. Isso quer dizer que, a magnitude das respostas dadas ao estímulo AVt é diretamente proporcional à magnitude das respostas dadas devido ao estímulo Ae. Por outro lado, as respostas dadas ao estímulo $\mathbf{V t}$ têm magnitude inversamente proporcional às dadas aos estímulos Ae e AVt. Isso significa que, quando o indivíduo ouve e vê a execução técnica faz uma associação resultante à audição da execução expressiva $(\mathrm{Ae})$. Porém, quando apenas vê, ele dá maior importância à execução técnica $(\mathrm{Vt})$ sem relacionar a uma audição resultante de execução expressiva. Veremos quais tipos de sensações relacionam-se predominantemente a estes estímulos ao associarmos as colunas aos eixos.

O segundo eixo fatorial (Eixo 2 em Ex.4) está caracterizado pelos estímulos Ve (que possui coordenada negativa), AVe (com coordenada positiva) At (com coordenada negativa), nesta ordem de importância para a geração desse eixo. Nota-se que, para esse eixo fatorial, existe correlação positiva entre os estímulos Ve e At e negativa entre esses e o estímulo AVe. As relações entre esses estímulos se dão como para o 
primeiro eixo fatorial. Tal fato nos indica que Ve e At provocam sensações semelhantes e distintas de AVe.

Tais caracterizações nos permitem tipificar o primeiro eixo fatorial como "do estímulo Ae e AVt". Os pontos correspondentes às colunas ${ }^{13}$ que tiverem coordenadas positivas no primeiro eixo corresponderão a sensações causadas preponderantemente por esses estímulos. O segundo eixo pode ser tipificado como "eixo associado ao GE (gesto expressivo)", sendo que os pontos correspondentes às sensações com sinal positivo em suas coordenadas do segundo eixo, serão causados preponderantemente pelo estímulo AVe; as sensações com sinal negativo no segundo eixo, terão sido causadas preponderantemente pelo estímulo Ve. Ou seja, o GE associa-se às sensações causadas pelos estímulos $\mathbf{A e}$ e Ve, enquanto o estímulo Ve, corresponderá às sensações associadas à parte negativa do segundo eixo.

5. Tipificação dos eixos fatoriais pelas colunas (sensações) - No que diz respeito à caracterização das colunas (as sensações expressas devidos aos estímulos) constatamos que, pelo Ex.5, as sensações de maior peso na formação do primeiro eixo fatorial foram de suspensão em F7, F8, F4, F3 e de conclusão em F4, com esta ordem de importância para a formação do primeiro fator.

\begin{tabular}{|l|l|}
\hline \multicolumn{2}{|l|}{ Contribuição Parcial } \\
\hline $\begin{array}{l}\text { Sensação } \\
\text { por fator (F) }\end{array}$ & Eixo 1 \\
\hline F7b & $\mathbf{1 6 , 3 9}$ \\
\hline F8b & $\mathbf{1 4 , 6 1}$ \\
\hline F4b & $\mathbf{1 4 , 5 2}$ \\
\hline F3b & $\mathbf{1 2 , 6 0}$ \\
\hline F4d & $\mathbf{1 0 , 1 5}$ \\
\hline F1d & 5,72 \\
\hline F3a & 3,95 \\
\hline F5b & 3,89 \\
\hline F3d & 3,11 \\
\hline F5a & 3,07 \\
\hline F7a & 3,07 \\
\hline F1a & 2,98 \\
\hline F2d & 1,76 \\
\hline F6a & 1,67 \\
\hline F2b & 0,87 \\
\hline F1b & 0,66 \\
\hline F2a & 0,35 \\
\hline F6b & 0,18 \\
\hline F7d & 0,16 \\
\hline F8d & 0,12 \\
\hline F6d & 0,08 \\
\hline F8a & 0,08 \\
\hline F4a & 0,02 \\
\hline Contbuca & \\
\hline
\end{tabular}

\begin{tabular}{|l|l|}
\hline \multicolumn{2}{|l|}{ Contribuição Parcial } \\
\hline $\begin{array}{l}\text { Sensação } \\
\text { por fator (F) }\end{array}$ & Eixo 2 \\
\hline F8d & $\mathbf{1 4 , 5 2}$ \\
\hline F2d & $\mathbf{1 3 , 5 7}$ \\
\hline F8a & $\mathbf{1 1 , 7 4}$ \\
\hline F5b & $\mathbf{1 1 , 6 6}$ \\
\hline F6a & $\mathbf{8 , 5 0}$ \\
\hline F6d & $\mathbf{8 , 5 0}$ \\
\hline F2b & 5,78 \\
\hline F1d & 4,07 \\
\hline F7d & 3,39 \\
\hline F4d & 2,62 \\
\hline F6b & 2,58 \\
\hline F3a & 2,42 \\
\hline F1b & 1,95 \\
\hline F2a & 1,85 \\
\hline F4a & 1,67 \\
\hline F8b & 1,64 \\
\hline F5a & 1,01 \\
\hline F7a & 1,01 \\
\hline F3b & 0,78 \\
\hline F3d & 0,66 \\
\hline F1a & 0,04 \\
\hline F4b & 0,01 \\
\hline F7b & 0,01 \\
\hline Fna \\
\hline
\end{tabular}

\begin{tabular}{|l|l|l|}
\hline \multicolumn{3}{|c|}{ Coordenadas } \\
\hline $\begin{array}{l}\text { Sensação } \\
\text { por fator (F) }\end{array}$ & Eixo 1 & Eixo 2 \\
\hline F1a & - & - \\
\hline F1b & - & - \\
\hline F1d & + & + \\
\hline F2a & - & + \\
\hline F2b & - & + \\
\hline F2d & - & - \\
\hline F3a & + & + \\
\hline F3b & - & - \\
\hline F3d & - & - \\
\hline F4a & + & + \\
\hline F4b & + & - \\
\hline F4d & + & - \\
\hline F5a & + & - \\
\hline F5b & - & + \\
\hline F6a & - & - \\
\hline F6b & - & + \\
\hline F6d & + & + \\
\hline F7a & + & - \\
\hline F7b & + & + \\
\hline F7d & - & - \\
\hline F8a & - & - \\
\hline F8b & - & + \\
\hline F8d & - & + \\
\hline
\end{tabular}

Ex.5 - Contribuição parcial das colunas (sensações) para a formação do $1^{\circ}$ e $2^{\circ}$ Eixos Fatoriais; sinais das coordenadas nos eixos fatoriais. 
Desde que o sinal de F7b, F4b e F4d é positivo constatamos correlação positiva com Ae e AVt que, assim, são os principais estímulos a causar essas sensações. Para o aparecimento dessas sensações o estímulo Vt teve muito pouca influência. Por outro lado, o sinal negativo das coordenadas das sensações F8b e F3b nesse primeiro eixo, indica que praticamente não existirá influência dos estímulos Ae e AVt para o seu aparecimento e que poderão sofrer leves influências do estímulo Vt.

O segundo eixo fatorial tem como principais responsáveis pela sua formação as sensações F8d, F2d, F8a, F5b, F6a e F6d, nessa ordem de importância. Desde que as sensações F8d, F5b e F6d têm suas coordenadas nesse segundo eixo positivas, estarão provocadas pelo estímulo AVe. Já, as sensações F6a, F2d e F8a que têm suas coordenadas, no segundo eixo, negativas foram provocadas pelo estímulo Ve.

6. Interpretação do gráfico - Tendo sido feitas as tipificações dos dois primeiros eixos fatoriais, segundo estímulos e caracterizadas as sensações que têm maior importância para o surgimento dos dois primeiros eixos fatoriais, ficamos habilitados para a interpretação do gráfico do primeiro plano fatorial, definido por esses dois eixos.

Antes, devemos observar que os pontos no gráfico são projeções no primeiro plano fatorial dos pontos originais definidos pela tabela de contingência. A AFC proporciona uma medida, chamada qualidade de representação do ponto no plano, definida pelo cosseno quadrado, que nos informa quão próximo ao plano está o ponto projetado. Essa medida varia de zero a um e nos informa que um ponto com qualidade de representação igual a 1, estará exatamente sobre o plano; quanto mais a qualidade de representação se aproxima de 0 , mais distante do plano estará o ponto. Tal informação é importante para que não haja confusão ao se estabelecer as relações entre pontos e eixos, na observação do gráfico desse plano. Quanto mais próxima de 1 for a qualidade de representação, mais firme será a relação entre o ponto e os eixos. A Tabela Ex.6 traz, em ordem decrescente, os valores para a qualidade de representação de linhas e colunas.

Vemos que, à exceção de Vt e At, os demais estímulos estão muito bem representados, mostrando boa correlação entre os mesmos e o plano. Isso significa que os pontos das sensações que tiverem boa qualidade de representação terão a influência desses estímulos, como já indicado. Assim, considerando a qualidade de representação das sensações no plano, ao observarmos o gráfico, poderemos dizer qual estímulo provoca tal sensação e como esta sensação se correlaciona com as demais.

Como exemplo, tomemos a sensação $\mathrm{F} 1 \mathrm{~d}$ que tem qualidade de representação igual a 0,9. Pela observação do gráfico da AFC (Ex.7), notamos que essa sensação se situa mais próxima do Eixo 2, pela parte positiva. Então, considerando a tipificação feita e a posição de F2a no gráfico (onde se situa em cima do Eixo 2 pela parte positiva), poderemos dizer que a sensação de continuidade em F2 foi devido ao estímulo AVe. 


\begin{tabular}{|l|l|}
\hline Estímulo & Qualidade \\
\hline Ae & 0,84 \\
\hline AVt & 0,84 \\
\hline Ve & 0,83 \\
\hline AVe & 0,71 \\
\hline Vt & 0,57 \\
\hline At & 0,54 \\
\hline
\end{tabular}

\begin{tabular}{|l|l|}
\hline $\begin{array}{l}\text { Sensação por } \\
\text { fator contextual } \\
\text { (F) }\end{array}$ & Qualidade \\
\hline F8a & 0,97 \\
\hline F2d & 0,93 \\
\hline F6d & 0,92 \\
\hline F5b & 0,92 \\
\hline F7b & 0,91 \\
\hline F1d & 0,90 \\
\hline F4b & 0,87 \\
\hline F4d & 0,85 \\
\hline F3b & 0,85 \\
\hline F1a & 0,82 \\
\hline F3a & 0,80 \\
\hline F8b & 0,79 \\
\hline F6a & $\mathbf{0 , 7 8}$ \\
\hline F8d & 0,66 \\
\hline F5a & $\mathbf{0 , 6 3}$ \\
\hline F7a & $\mathbf{0 , 6 3}$ \\
\hline F2a & $\mathbf{0 , 5 9}$ \\
\hline F7d & $\mathbf{0 , 5 4}$ \\
\hline F6b & $\mathbf{0 , 5 1}$ \\
\hline F2b & $\mathbf{0 , 5 1}$ \\
\hline F1b & 0,40 \\
\hline F3d & 0,21 \\
\hline F4a & 0,20 \\
\hline
\end{tabular}

Ex.6 - Qualidade de representação para os estímulos e para as sensações no primeiro plano fatorial.

Outro exemplo poderá ser a leitura feita sobre F8a que tem a melhor qualidade de representação $(0,97)$, mas aparece pela parte negativa do Eixo 1. Ao considerarmos a mesma tipificação e posição de F6a no gráfico, percebemos como esse fator surge próximo ao estímulo Ve, atestando a influência desse estímulo sobre a sensação de continuidade em F6: 


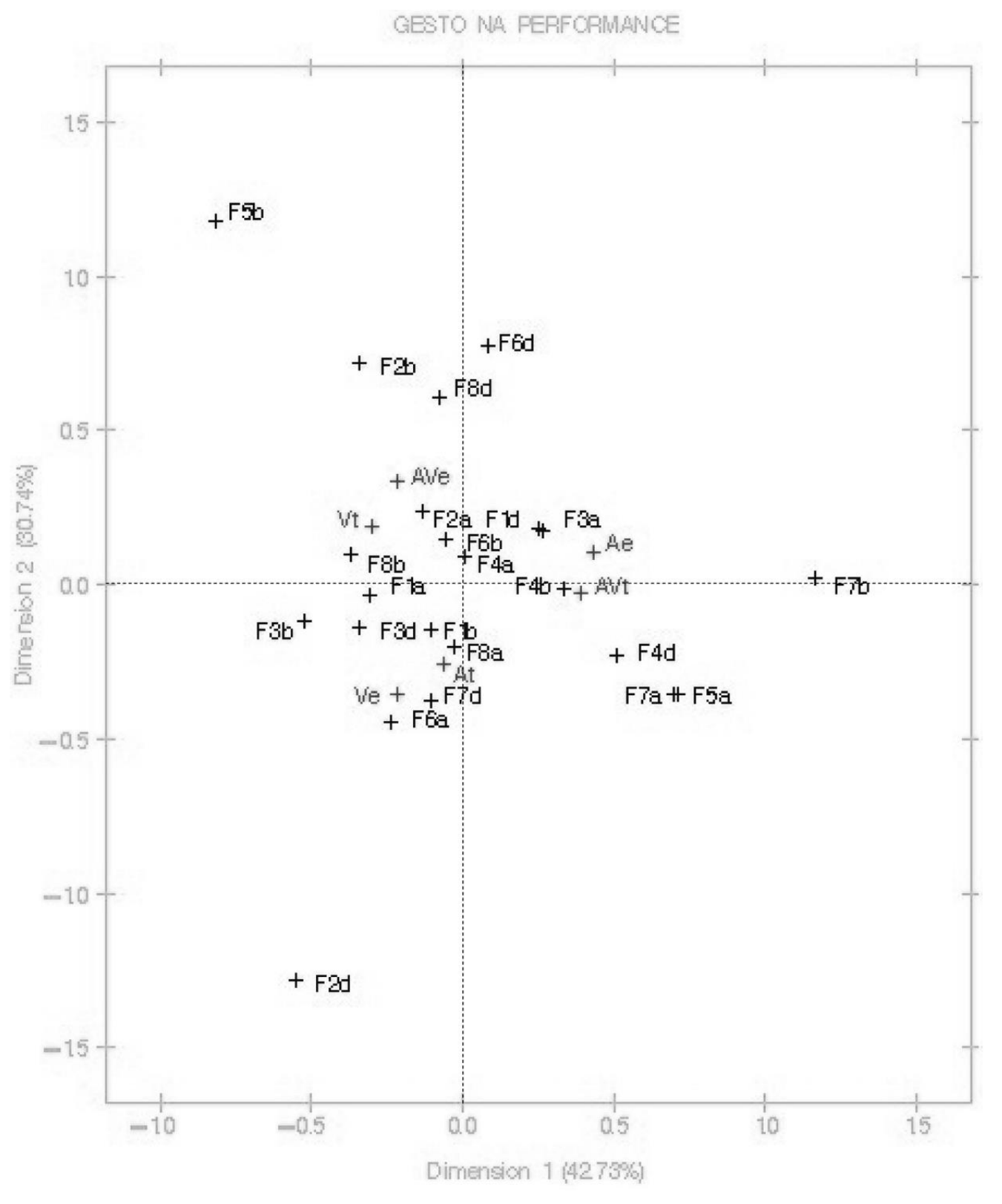

Ex.7 - Gráfico da AFC, onde: Dimension 1 = Eixo 1. Dimension 2 = Eixo 3.

Podemos dizer que as influências sofridas pelas sensações nos Fatores Contesxtuais (F) variaram de estímulo para estímulo, sendo que $\mathbf{A e}$ e AVt tiveram mais peso nas sensações próximas ao Eixo 1 e Ve e AVe nas sensações próximas ao Eixo 2. Independentemente de ser GT ou GE, o estímulo audiovisual (AV) foi o que surgiu com maior peso nos dois eixos, em relação a $\mathbf{A}$ e $\mathbf{V}$, tendo maiores influências nas percepções das sensações de continuidade, suspensão e conclusão. Tratando-se propriamente de música, era de se esperar que o estímulo $\mathrm{A}$ se evidenciasse, porém é realmente relevante o resultado referente ao estímulo $\mathbf{V}$. Percebemos que, por esse prisma, o aspecto visual não pode ser ignorado na performance destinada à música para percussão. 
É natural que, sobre esse ponto de vista e através dos elementos aqui apresentados, constatemos que todos os tipos de estímulos ( $\mathbf{A}, \mathbf{V}$ e $\mathbf{A V}$ ) e de atitudes do gesto (GE e GT) presentes no experimento causaram, sob distintos níveis, influência nas sensações de continuidade, suspensão e conclusão a cerca dos trechos executados.

Nos resultados da AFC a expressividade da execução, representada por GE, figurou com maior peso de influência representado pelos estímulos $\mathbf{A}, \mathbf{V}$ e AV. Já o GT surge como agente influenciador através do estímulo AV (os estímulos V e A tiveram pouco peso e menores níveis de influência aliados ao GT). Seguindo essa perspectiva e através daquilo que buscamos ilustrar relativamente às questões do GE e GT, percebemos uma variação das influências em relação às três sensações do experimento, destoando levemente daquilo que ocorreu na APD (CHAIB et. al. 2013). Como exemplo podemos citar F1 que tem, na AFC, a sensação de conclusão com maior influência a partir do estímulo AVe.

Os estímulos AVe e Ve condisseram com o sentido de continuidade nos fatores $\mathrm{F} 2$ e F6. O GE também surge como agente influenciador em F3, para a sensação de continuidade (sendo neste caso o estímulo V). Para F4 por exemplo, a sensação de suspensão obteve maior influência em AVt. A sensação de continuidade em F4 também teve maior peso através desse mesmo estímulo. Desta feita afirmamos que, sendo GT ou GE, a visualização dos movimentos corporais (estando em convergência ou não com o estímulo auditivo) exerceram influência sobre as sensações de continuidade, suspensão e conclusão dos participantes do experimento sobre uma performance percussiva.

\section{Impressões e conclusões sobre as análises realizadas -}

Do que pudemos analisar, será difícil afirmar concretamente ou de uma maneira mais cartesiana que o GE em conjunção com os estímulos influenciará sempre a um nível preciso de sensação e o $\mathbf{G T}$, da mesma forma, em outro nível. Essas duas possibilidades de movimentos corporais parecem causar influência sobre as sensações de continuidade, suspensão e conclusão, não apenas quando estão aliadas aos estímulos apresentados, mas também aos contextos sonoros e musicais em questão (timbre, meios expressivos, duração, etc.). Daí a impossibilidade de "taxar" ou de "rotular" os diferentes meios de movimentos a qualquer que seja a sensação atribuída na performance em percussão.

De certa forma justifica-se, através do "Gesto Percussivo Interpretativo" (CHAIB, 2013, p.165), o trabalho intelectual do músico percussionista em palco. Se, hipoteticamente, gestos e atitudes específicos (no caso aqui GE e GT) influenciassem sempre os mesmos juízos de valores, a busca por uma expressividade própria do músico passaria a ser obsoleta e desnecessária. O seu desempenho enquanto performer limitar-se-ia às questões técnicas do instrumento, uma vez que seus movimentos já seriam a própria linha de transmissão de toda mensagem a ser passada, interpretativamente e expressivamente, para a audiência. Se o GE e GT possuíssem uma acepção especifica e invariável sobre as sensações aqui estudadas, bastaria ao intérprete seguir um método de movimentos pré-estabelecidos para garantir, em parte, o sucesso da transmissão das mesmas. 
A AFC assegura-nos que essas relações das respostas poderão se manter, caso outros estudos sejam realizados sobre os mesmos parâmetros de performance. Isso nos possibilita atestar a inegável influência que o aspecto visual causa nas sesnações do espectador sobre a performance percussiva, estando em convergência com o estímulo auditivo (em forma de estímulo $\mathbf{A V}$ ) ou de forma isolada (como apenas estímulo V).

Constatada a influência que os gestos podem causar sobre as sensações de continuidade, suspensão e conclusão a partir da execução de um trecho musical realizada por um percussionista consideraremos, o movimento corporal, uma das principais fontes de expressividade para a elaboração da sua performance. Acreditamos que o desenvolvimento e a percepção dos gestos potencialmente exequíveis durante a execução instrumental percussiva permitirá o alargamento das possibilidades de transmissão do conteúdo musical da obra reproduzida pelo intérprete. Isso poderá gerar diferentes níveis de expressão levando o performer a ampliar as suas faculdades interpretativas, possibilitando ao espectador experimentar novos sentidos de interpretação e expressão, ilustrados em palco pelo percussionista.

Através de toda essa reflexão diremos que o intérprete percussionista poderá usufruir dos conceitos de "movimento corporal" através de diferentes atitudes gestuais (dentre elas o GE e GT); "memória corporal"; "tipologias de gesto" em percussão (Gesto Percussivo, Gesto Percussivo Interpretativo e Gesto Percussivo Expressivo). Tais conceitos são desenvolvidos e especificados no artigo "Três Perspectivas Gestuais para uma Performance Percussiva: Técnica, Interpretativa e Expressiva" (CHAIB, 2013). No nosso entender trata-se, antes de mais nada, de legitimar uma preocupação do uso do corpo no fazer musical inserida nessa performance, sem qualquer intenção coreográfica ou de construção de uma espécie de mise en cène sobre 0 ato performativo. Buscar compreender as características musicais da obra e, a seguir, transpô-las para os movimentos corporais (segundo as suas próprias ideias) parece ser um bom começo para a elaboração dessa relação gestual entre a música e o corpo.

Enfatizamos que a elaboração gestual corporal do percussionista deverá ser sempre em função da obra, e não o contrário. A música, o seu resultado sonoro enquanto obra musical deverá sempre ser o mais importante. Para nós, o corpo deverá ser um auxiliário na transmissão das ideias musicais. Caso contrário corre-se o risco de a performance tornar-se excessivamente cênica inapropriada para o tipo de performance musical que aqui estudamos. O resultado musical final poderá mesmo ser prejudicado caso o trabalho sobre a música em si seja posto em segundo plano, ou seja, passe a estar em função dos movimentos corporais.

Partimos do princípio de que a preocupação com o corpo deva existir a partir do momento em que a obra a ser interpretada já esteja dominada. Ou seja, onde a inserção do trabalho corporal numa perspectiva gestual apenas ampliará ou auxiliará nas capacidades comunicativas da performance e/ou da obra. Isso, efetivamente, traz à tona questões éticas, entre elas a honestidade do performer para com o público no ato de executar uma obra musical. Para nós, a valorização do trabalho realizado com o corpo, conjuntamente com a composição dos seus movimentos, significa gerar uma concepção performativa percussiva preocupada em congregar os diversos elementos 
atuantes em palco, frutos da reprodução de uma performance musical. O gesto será, sem dúvida, um deles.

\section{Referências}

BATISTA, J.M., e J. SUREDA (1987). Análisis de correspondencias y técnicas de clasificación: Su interés para la investigación en las ciencias sociales y del comportamiento. Infancia y Aprendizaje n.39/40: 171-186.

BARROSO, L. P. (2003). Analise Multivariada. Colóquio na 48a Reunião da RBRAS e 10o SEGRO. Lavras..

CHAIB, F. (2013) "Três Perspectivas Gestuais para uma Performance Percussiva: Técnica, Interpretativa e Expressiva". Per Musi 27, p.159-181. Belo Horizonte.

(2012) O Gesto na Performance em Percussão: Uma Abordagem Sensorial e Performativa. Tese de Doutorado. Departamento de Comunicação e Arte da Universidade de Aveiro. Aveiro.

CHAIB, F. J. CATALÃO, H. CHAIB FILHO (2013) "A Influência do Gesto na Performance em Percussão: Análise Descritiva de Dados Experimentais". Per Musi ???. Belo Horizonte.

LEBART, L.; A. MORINEA e M. PIRON. (2002). Statistique exploratoire multidimensionnelle. 3a ed. Paris: Dunod.

SANTIAGO, P. e A. MEYEREWICZ (2009). "Considerações piercinanas sobre o gesto na performance do Grupo UAKTI". Per Musi n.20: 83-91. Belo Horizonte.

\section{Literatura recomendada}

DAHL, S. (2000). "The Playing of an Accent - Preliminary Observations from Temporal and Kinematic Analysis of Percussionists". Journal of New Music Research n.29 vol.3: 225-233.

(2004). "Playing the Accent - Comparing Striking Velocity and Timing in an Ostinato Rhythm

Performed by Four Drummers". Acta Acustica n.90: 762-776

(2005). On the beat: Human movement and timing in the production and perception of music. $\mathrm{PhD}$ Thesis, KTH Computer Science and Communication, KTH School of Computer Science and Communication, Stockholm.

GODØY, R. 2011. "Coarticulated Gestural-sonic Objects in Music". In. New Perspectives in Music and Gesture. SEMPRE. MPG Books Group. UK.

GODØY, R., e M. LEMAN. (2010). Musical Gestures - Sound, Movement, and Meaning. NewYork: Routledge.

SCHUTZ, M. e S. LIPSCOMB. (2004). "Influence of Visual Information On Auditory Perception Of Marimba Stroke Types." ANAIS do VIII International Conference of Music Perception and Cognition (ICMPC8), s/p. Evanston.

. 2007. "Hearing gestures, seeing music: Vision influences perceived tone duration". Perception n.36: 888-897.

SMITH, S. A. (1941) Suite for Drums. New York: Fillmore Music House.

WANDERLEY, M., B. VINES, N. MIDDLETON, C. MACKAY, e W. HATCH. (2005). "The Musical Significance of Clarinetists' Ancillary Gestures: An Exploration of the Field". Journal of New Music Research n.34: 97-113.

Fernando Chaib é Doutor e Mestre em Música/Performance pelo Departamento de Comunicação e Arte da Universidade de Aveiro (Portugal) e graduou-se em percussão pela UNESP. Possui prêmios como solista e camerísta em países como Itália, Portugal e Brasil. Vem atuando nos continentes asiático, europeu e americano. Tem artigos nos principais periódicos especializados em música no Brasil e em congressos no exterior. Atualmente é professor no Instituto Federal de Goiás, onde coordena o Núcleo de Excelência para o Ensino, Pesquisa e Performance em Percussão - N三P/IFG (que integra o Centro de Excelência para o Ensino, Pesquisa e Performance em Percussão $\left.C \equiv P^{3}\right)$.

Homero Chaib Filho é Doutor em Matemática Aplicada pela Escuela Técnica Superior de Ingenieros Agrónomos de la Universidad de Madrid (Espanha), além de pesquisador 
aposentado pela EMBRAPA. Realizou comunicações em congressos nos continentes europeu, asiático e americano. Possui publicações nacionais e internacionais.

João Catalão é Doutor em Música/Performance pela Universidade de Montreal (Canadá), tendo se especializado no Conservatório de Estrasburgo (França) e graduado na UFSM. É membro dos grupos de percussão KT-Z (Brasil) e Sixtrum (Canadá). Toca em países no continente europeu e americano.

\footnotetext{
1 Este artigo é parte do Capítulo 3 da Tese de Doutorado de Fernando Chaib intitulada $O$ Gesto na Performance em Percussão: Uma Abordagem Sensorial e Performativa, defendida no Departamento de Comunicação e Arte da Universidade de Aveiro. Aveiro.

2 Núcleo de Excelência para o Ensino, Pesquisa e Performance em Percussão do Instituto Federal de Educação, Ciência e Tecnologia de Goiás.

${ }^{3}$ Núcleo de Excelência para o Ensino, Pesquisa e Performance em Percussão do Instituto Federal de Educação, Ciência e Tecnologia de Goiás.

${ }^{4}$ Visual (V), Áudio (A) e Audiovisual (AV).

${ }^{5}$ Gesto Técnico (GT) e Gesto Expressivo (GE).

${ }^{6}$ Como descrito no artigo "A Influência do Gesto na Performance em Percussão: Análise Descritiva de Dados Experimentais" (CHAIB et. al. 2013), os Fatores Contextuais significaram "situações que acreditamos 'desestabilizar' um sentido de continuidade (por exemplo, situações de 'ruptura', 'quebra', 'tensão', etc.)". Por exemplo: mudanças bruscas de dinâmica; pausas; fermatas; mudanças bruscas de timbres; etc.

${ }^{7}$ A metodologia utilizada para a realização desse experimento poderá ser consultada na íntegra no artigo "A Influência do Gesto na Performance em Percussão: Análise Descritiva de Dados Experimentais" (CHAIB et. al. 2013).

${ }^{8}$ Tabela extraída do artigo "A Influência do Gesto na Performance em Percussão: Análise Descritiva de Dados Experimentais" (CHAIB et. Al. 2013).

9 Não confundir com "Fatores Contextuais".

${ }^{10}$ Cujo tratamento teórico foi devido a Benzécri (LEBART et al. 2000, p.67).

11 Ver gráfico da AFC (Ex.7).

12 Deste ponto em diante trataremos as linhas por estímulos.

${ }^{13}$ Deste ponto em diante trataremos as colunas como sensações.
} 